\title{
Induced Magnetic Monopole from Trapped $\Lambda$-Type Atom
}

\author{
P. Zhang, Y. Li and C. P. Sun ${ }^{a, b}$ \\ Institute of Theoretical Physics, the Chinese Academy of Sciences, Beijing, 100080, China
}

\begin{abstract}
We investigate the spatial motion of the trapped atom with the electromagnetically induced transparency (EIT) configuration where the two Rabi transitions are coupled to two classical light fields respectively with the same detuning. When the internal degrees of freedom can be decoupled adiabatically from the spatial motion of the center of mass via the Born-Oppenheimer approximation, it is demonstrated that the lights of certain profile can provide the atom with an effective field of magnetic monopole, which is the so-called induced gauge field relevant to the Berry's phase. Such an artificial magnetic monopole structure manifests itself in the characterizing energy spectrum.
\end{abstract}

PACS number:03.65.Vf,42.50.Vk,14.80.Hv

Modern concept of magnetic monopole in quantum mechanics was postulated by Dirac in 1931 [1]. Since that time physicist have been making efforts to seek for the magnetic monopole in real space for more than seventy years. Though convincing evidence for its existence in real space has not yet been found, the theoretical conception of magnetic monopole has initiated many important progresses in both physics and mathematics. In fact, in the extremely high energy scale that we have not reached at present, the grand unification theory [2] predicted the magnetic monopole as a consequence of the beautiful topology structure of Yang-Mills theory [3]. The discovery of Berry phase also resulted in a physical implementation of magnetic monopole in the parameter space [4]. Precisely speaking, the degeneracy point in the parameter space acts like a magnetic monopole caused by an effective gauge field. The Berry phase based magnetic monopole of this kind can be demonstrated in association with the anomalous Hall effect of ferromagnetic metals [5] where the slowly changing parameter is just the crystal momentum. Now we consider an artificial realization of magnetic monopole in real space.

For the quantum adiabatic process induced Berry phase, it is well known that when the slowly varying parameters are the dynamic variables of a subsystem interacting with another subsystem with fast varying variables, the adiabatic separation of the two subsystems via the Born-Oppenheimer approximation [7] can provide the slow subspace with a scaler and a vector potentials [6] called the induced gauge potential. In the neutron spin precession experiment [8] the Aharonov-Borhm effect caused by this vector potential was pointed out as a manifestation of Berry phase. In this article, we will derive the monopole type induced gauge field for the spatial motion of $\Lambda$-type atom interacting with control and probe laser beams which drive the transitions $|e\rangle-|1\rangle$ and $|e\rangle-|2\rangle$ respectively (see Fig. 1) [9,10]. We will also show that when the atom is cold enough, its internal degrees of freedom can decouple adiabatically from the spatial motion of the center of mass. Correspondingly, the BornOppenheimer approximation provides the atomic center of mass with an effective magnetic monopole field as a special induced gauge field relevant to the Berry phase when the lights are artificially shaped in certain profiles. We predict that such an artificial magnetic monopole can be observed experimentally through its special spectral structure. In fact, the prompt advance in experiments of trapping and cooling atoms has provided a platform to test our predictions exactly.

The Hamiltonian for our cold atom system driven by two laser beams (see Fig. 1) can be written in the form $H=\mathbf{P}^{2} / 2 M+H_{f}(\mathbf{r})$ with the local internal Hamiltonian

$$
\begin{aligned}
H_{f}(\mathbf{r})= & \sum_{i=1}^{2} V_{i}(\mathbf{r})|i\rangle\langle i|+\Delta| e\rangle\langle e| \\
& +\Omega_{p}(\mathbf{r})|e\rangle\left\langle 1\left|+\Omega_{c}(\mathbf{r})\right| e\right\rangle\langle 2|+\text { h.c.. }
\end{aligned}
$$

Here, $\mathbf{r}$ is the atomic position, $\Delta$ the one-photon detunning, $\Omega_{c}(\mathbf{r})\left(\Omega_{p}(\mathbf{r})\right)$ the Rabi frequency of the probe (control) beam, and $V_{i}(\mathbf{r})$ the trap potential of the $i$-th inner energy level. We assume that there is no trap for the level $e$, i.e., $V_{e}(\mathbf{r})=0$, and there is the same trap for both level 1 and 2, i.e., $V_{1}(\mathbf{r})=V_{2}(\mathbf{r})=V(\mathbf{r})$. This assumption just ensures the occupance of the dark state.

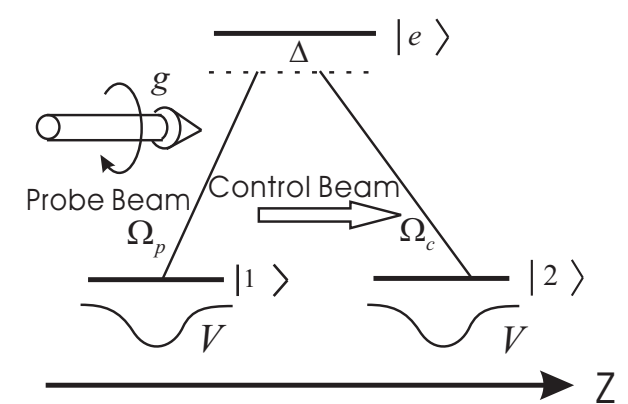

FIG. 1. Three level atoms interacting with two laser beams. The probe beam coupling the states $|e\rangle$ and $|1\rangle$ has an orbital angular momentum. The atoms in state $|1\rangle$ and $|2\rangle$ are trapped by the potential $V$, which is a function of the atomic position $\mathbf{r}$.

To use the generalized Born-Oppenheimer approximation [8], we first diagonalize the interaction part $H_{f}(\mathbf{r})$ of 
the Hamiltonian and obtain the $\mathbf{r}$-dependent eigenvalues of $H_{f}(\mathbf{r}): E_{0}(\mathbf{r})=V(\mathbf{r})$ and

$$
E_{ \pm}(\mathbf{r})=\frac{1}{2}\left(\tilde{\Delta} \pm \sqrt{4\left|\Omega_{c}\right|^{2}+4\left|\Omega_{p}\right|^{2}+\tilde{\Delta}^{2}}\right)+V(\mathbf{r}) .
$$

where $\tilde{\Delta}=\tilde{\Delta}(\mathbf{r})=\Delta-V(\mathbf{r})$ is the local one-photon detunning. The eigenstate corresponding to $E_{0}(\mathbf{r})$ is the r-dependent dark sate defined as

$$
|D(\mathbf{r})\rangle=\frac{1}{\Omega}\left[\Omega_{p}|2\rangle-\Omega_{c}|1\rangle\right] .
$$

where $\Omega=\Omega(\mathbf{r})=\sqrt{\left|\Omega_{c}\right|^{2}+\left|\Omega_{p}\right|^{2}}$. The other two eigenstates corresponding to the eigenenergies $E_{ \pm}(\mathbf{r})$ can be noted as $\left|B_{ \pm}(\mathbf{r})\right\rangle$. Their explicit expressions are not necessary to the following discussion for the case that the atom spatial motion is sufficiently slow so that the internal motion is not excited.

It is well known that the atomic wave function in the r-representation can be written as

$$
\langle\mathbf{r} \mid \Psi\rangle=\psi_{0}(\mathbf{r})|D(\mathbf{r})\rangle+\sum_{k=+,-} \psi_{k}(\mathbf{r})\left|B_{k}(\mathbf{r})\right\rangle .
$$

When the energy gaps $E_{ \pm}-E_{0}(\mathbf{r})$ between the dark state $|D(\mathbf{r})\rangle$ and the states $\left|B_{ \pm}(\mathbf{r})\right\rangle$ are large enough, the Born-Oppenheimer approximation is applicable. Under this approximation, the atom can be assumed to be "kept" in the dark state $|D(\mathbf{r})\rangle$ at every position $\mathbf{r}$ and the eigen wave function of the total Hamiltonian $H$ can be written as $\langle\mathbf{r} \mid \Psi\rangle=\psi_{0}(\mathbf{r})|D(\mathbf{r})\rangle$ where $\psi_{0}(\mathbf{r})$ satisfies the eigenequation

$$
\left[\frac{1}{2 M}\left(\mathbf{P}+\mathbf{A}_{0}\right)^{2}+V(\mathbf{r})\right] \psi_{0}(\mathbf{r})=E \psi_{0}(\mathbf{r}) .
$$

Here, $\mathbf{A}_{0}(\mathbf{r})=-i\langle D(\mathbf{r})|\nabla| D(\mathbf{r})\rangle$ is the induced gauge potential corresponding to the dark state.

As in Ref. [12], we may assume that the probe beam and the control beam have the same frequency and propagate along the $z$ direction. Then the Rabi frequencies $\Omega_{p}(\mathbf{r})$ and $\Omega_{c}(\mathbf{r})$ can be expressed as

$$
\begin{aligned}
& \Omega_{p}=\left|\Omega_{p}\right| \exp (i \mathbf{k} \cdot \mathbf{r}+g \phi), \\
& \Omega_{c}=\left|\Omega_{c}\right| \exp (i \mathbf{k} \cdot \mathbf{r}) .
\end{aligned}
$$

Here, the real parameters $\left|\Omega_{p}(\mathbf{r})\right|$ and $\left|\Omega_{c}(\mathbf{r})\right|$ are just the slowly varying norms of the $\Omega_{p}$ and $\Omega_{c}$ and $\phi$ the directional angle of the $x-y$ plane. Note that in writing down Eq. (6), we have also assumed that the probe beam has an orbital angular momentum $g \phi$ with $g$ an integer [11]. Then the induced gauge potential $\mathbf{A}_{0}$ of the dark state can be written as

$$
\mathbf{A}_{0}=\nabla \phi_{c}+\frac{g\left|\Omega_{p}\right|^{2}}{\Omega^{2}} \nabla \phi
$$

It is obvious that the orbital angular momentum number $g$ of the probe beam induces an effective magnetic monopole of strength $g$. We thus conclude that the effective magnetic charge of this induced monopole can be controlled artificially by adjusting the angular momentum of photons in the probe beam.

When the norms $\left|\Omega_{p}(\mathbf{r})\right|$ and $\left|\Omega_{c}(\mathbf{r})\right|$ of the Rabi frequencies take the forms $\left|\Omega_{p}\right|^{2}=\xi(r+z),\left|\Omega_{c}\right|^{2}=$ $\xi(r-z)$, the potential $\mathbf{A}_{0}$ has the same form as that of the potential created by a monopole and can be expressed as

$$
\mathbf{A}_{0}=\nabla \phi_{c}+\frac{g(1+\cos \theta)}{2 r \sin \theta} \mathbf{e}_{\phi} .
$$

Here, $r, \theta$ and $\phi$ are just the spherical polar coordinates. In this case we have $\left|\Omega_{p}\right|=\left|\Omega_{c}\right|=0$ at the origin $\mathbf{r}=0$. This leads to an "accidental degeneracy" $E_{ \pm}(\mathbf{0})=E_{0}(\mathbf{0})$ at the origin where the energy levels cross. Thus the Born-Oppenheimer approximation does not work well. For this reason, in the following discussion, we only discuss the atomic motion around the region far away from the origin.

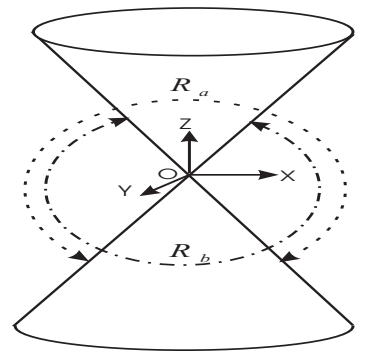

FIG. 2. The whole space excluding the origin $O$ is divided into two regions $R_{a}$ and $R_{b} . R_{a}$ is the space excluding the lower circular cone (the region enveloped by the dashed line), $R_{b}$ is the space excluding the upper one (the region enveloped by the dash dot line). The space outside of the two cones is the overlap of $R_{a}$ and $R_{b}$.

For the original Dirac's monopole, as is well known, the induced monopole potential $\mathbf{A}_{0}$ has singularity at the string of $\theta=0$. But this singularity can be exorcized by means of the approach developed by Wu and Yang $[3,13]$. To this end one needs to divide the total real space (except the origin) into two regions (see Fig. 2), $R_{a}: 0 \leq \theta<\frac{\pi}{2}+\delta$ and $R_{b}: \frac{\pi}{2}-\delta \leq \theta<\pi$, which are two overlapping caps and have their well defined local coordinates. Then the dark state $|D(\mathbf{r})\rangle$ and the wave function $\psi_{0}(\mathbf{r})$ can no longer be defined globally. Instead, $|D(\mathbf{r})\rangle$ and $\psi_{0}(\mathbf{r})$ will have different expressions in the different regions marked by $a$ and $b$. For instance the dark state can be defined as

$$
|D(x)\rangle_{a}=e^{-i\left(\phi_{c}+g \phi\right)} \frac{1}{\Omega}\left(\Omega_{p}|2\rangle-\Omega_{c}|1\rangle\right)
$$

in $R_{a}$ and 


$$
|D(x)\rangle_{b}=e^{-i \phi_{c}} \frac{1}{\Omega}\left(\Omega_{p}|2\rangle-\Omega_{c}|1\rangle\right)
$$

in $R_{b}$. The Schroedinger equation (5) can be rewritten in different caps as

$$
\left[\frac{1}{2 M}\left(\mathbf{P}+\mathbf{A}_{0 \alpha}\right)^{2}+V(\mathbf{r})\right] \psi_{0 \alpha}(\mathbf{r})=E \psi_{0 \alpha}(\mathbf{r})
$$

in $R_{\alpha}$ for $\alpha=a, b$. Here, $\psi_{0 a}(\mathbf{r})$ and $\psi_{0 b}(\mathbf{r})$ are the expressions of $\psi_{0}(\mathbf{r})$ in $R_{a}$ and $R_{b}$ respectively and the gauge potential $\mathbf{A}_{0 a}\left(\mathbf{A}_{0 b}\right)$ can be expressed as

$$
\mathbf{A}_{0 a}=\frac{g(-1+\cos \theta)}{2 r \sin \theta} \hat{e}_{\phi}, \quad \mathbf{A}_{0 b}=\frac{g(1+\cos \theta)}{2 r \sin \theta} \hat{e}_{\phi} .
$$

Apparently, $\mathbf{A}_{0 a}\left(\mathbf{A}_{0 b}\right)$ is not singular in the region $R_{a}$ $\left(R_{b}\right)$. In the overlap of $R_{a}$ and $R_{b}$, we have a connection $\psi_{0 b}(\mathbf{r})=\psi_{0 a}(\mathbf{r}) e^{-i g \phi}$ due to the $U(1)-$ guage. Such two local wave functions in two caps with this connection in the overlapping region are mathematically called the wave section.

We now consider a simple case that the trap potential $V(\mathbf{r})$ is spherically symmetrical. In this case we can separate the radical degree of freedom $r$ and the angular degrees of freedom $\theta$ and $\phi$ in the Schroedinger equation (11) by invoking the generalized angular momentum operator [13]

$$
\mathbf{L}=\mathbf{r} \times\left(\mathbf{P}+\mathbf{A}_{0}\right)-\frac{g \mathbf{r}}{2 r} .
$$

It was proved [13] that $\psi_{0}(\mathbf{r})=R_{l}(r) Y_{q, l, m}$ can be expressed in terms of the monopole harmonics $Y_{\frac{g}{2}, l, m}(\theta, \phi)$ which is the common "eigensection" of $L^{2}$ and $L_{z}$ with respect to the eigenvalues $l(l+1)$ and $m$. Here, we have $l=\left|\frac{g}{2}\right|,\left|\frac{g}{2}\right|+1, \ldots$ and $m=-l,-l+1, \ldots, l$. The radical wave function $R_{l}(r)$ satisfies the equation

$$
\left[-\frac{\partial_{r}\left(r^{2} \partial_{r}\right)}{2 M r^{2}}+\frac{l(l+1)-(g / 2)^{2}}{2 M r^{2}}+V(r)-E\right] R_{l}=0 .
$$

In the special case $V=0$, when $E>0$, the solution of Eq. (14) is [14] a Bessel function $R=\frac{1}{\sqrt{k r}} J_{\mu}(k r)$ with

$$
\mu=\sqrt{l(l+1)-\left(\frac{g}{2}\right)^{2}+\frac{1}{4}}, \quad k=\sqrt{2 M E} .
$$

Next we consider a more interesting case. We assume the the trap is a harmonic potential

$$
V=\frac{1}{2} M \omega_{z}^{2}\left(z-z_{0}\right)^{2}+\frac{1}{2} M \omega^{2} \rho^{2}
$$

where $z_{0}>0$ and $\rho^{2}=x^{2}+y^{2}$ (see Fig. 3). In this case, the atom is confined near a fixed point $\left(0,0, z_{0}\right)$ in the region $R_{a}$. Therefore, we need only consider the expression $\psi_{0 a}$ of $\psi_{0}$ in $R_{a}$. Since the trap potential is cylindrically symmetrical, it is convenient to discuss this problem with the cylindrical coordinate $(\rho, z, \phi)$. Because of the cylindrical symmetry of $V$ and $\mathbf{A}_{0}$, the wave function $\psi_{0 a}$ has the factor $\exp (i m \phi)(m=0, \pm 1, \pm 2, \ldots)$ which is just the generator of the rotation along $z$ axis. Then we have $\psi_{0 a}=T_{m}(\rho, z) \exp (i m \phi)$ where $R_{m}(\rho, z)$ satisfies the radical Schroedinger equation

$$
\frac{1}{2 M}\left[-\partial_{\rho}^{2}-\frac{1}{\rho} \partial_{\rho}-\partial_{z}^{2}+F_{m}(\rho, z)\right] T_{m}+V T_{m}=E T_{m} .
$$

Here, the function $F_{m}(\rho, z)$ is defined as

$$
F_{m}(\rho, z)=\left(\frac{m}{\rho}+g \frac{z-\sqrt{\rho^{2}+z^{2}}}{2 \sqrt{\rho^{2}+z^{2}} \rho}\right)^{2} .
$$

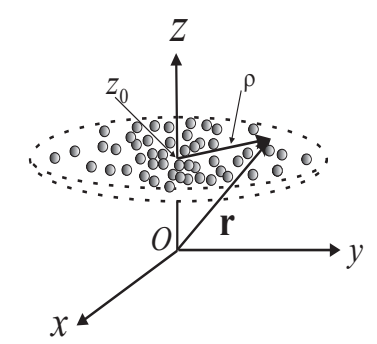

FIG. 3. The atoms are trapped in the "discus form" region whose center is the point $\left(0,0, z_{0}\right)$. In this figure, $O$ is the origin of the coordinate.

If the trap along $z$ axis is strong enough, i.e., $\omega_{z}$ is large enough, we can make the approximation $F_{m}(\rho, z) \approx$ $F_{m}\left(\rho, z_{0}\right)$. It is obvious that $F_{m}\left(\rho, z_{0}\right)$ can be expanded as a Laurent series of $\rho$. We also assume that the trap localized in $x-y$ plane is also strong enough that we can only keep $F_{m}\left(\rho, z_{0}\right)$ up to the term proportional to $\rho^{2}$. Thus approximately we have

$$
F_{m}(\rho, z) \approx \frac{m^{2}}{\rho^{2}}+\frac{g^{2}}{16 z_{0}^{4}} \rho^{2}-\frac{m g}{2 z_{0}^{2}},
$$

and we can solve the Eq. (17) to obtain the energy spectrum

$$
E_{m, n_{\rho}, n_{z}}=\left(2 n_{\rho}+|m|+1\right) \widetilde{\omega}-\frac{m g}{4 M z_{0}^{2}}+\left(n_{z}+\frac{1}{2}\right) \omega_{z}
$$

in terms of the radical quantum number $n_{\rho}$ and the vertical one $n_{z}(=0,1,2, \ldots)$, where

$$
\widetilde{\omega}=\sqrt{\omega^{2}+\frac{g^{2}}{16 M^{2} z_{0}^{4}}}
$$

is the modified radical frequency for the two dimensional reduced radical oscillator. The corresponding radical frequency shift $\widetilde{\omega}-\omega \sim-\frac{m g}{4 M z_{0}^{2}}$ can be regarded as the first 
observable effect of the artificial magnetic monopole. The additional term $-\frac{m g}{4 M z_{0}^{2}}$ in the energy spectrum of the spatial motion of atom may reflect its effect in realistic experiment. The corresponding wave function can also be obtained explicitly:

$$
\begin{aligned}
\psi_{0 a}^{m, n_{\rho}, n_{z}}= & N_{n_{z}} e^{i m \phi} \rho^{|m|} e^{-M\left(\widetilde{\omega}+\omega_{z}\right) \rho^{2} / 2} \\
& \times F\left(-n_{\rho},|m|+1, M \widetilde{\omega} \rho^{2}\right) H_{n_{z}}\left(\sqrt{M \omega_{z}} z\right) .
\end{aligned}
$$

Here, $N_{n_{z}}=\left[\sqrt{M \omega_{z}} / \sqrt{\pi} 2^{n_{z}} n_{z} !\right]^{\frac{1}{2}}, F$ is the confluent hypergeometric function and $H_{n_{z}}$ the Hermit function.

Since the above results are achieved with the generalized Born-Oppenheimer approximation, we should investigate the condition under which this approximation is applicable. The adiabatic condition can be obtained semiclassically. For simplicity, we only consider the case $\Delta=0$. In our problem, the sufficient condition of adiabatic approximation is

$$
\left|\left\langle D|\nabla| B_{ \pm}\right\rangle \cdot \mathbf{v}\right| \cdot\left|E_{ \pm}-E_{0}\right|^{-1}<<1
$$

where $\mathbf{v}$ is the velocity of the atomic center of mass (c.m). By straightforward calculation, it can be obtained that the upper limit of $\left|\left\langle D|\nabla| B_{ \pm}\right\rangle \cdot \mathbf{v}\right|$ is $\frac{1}{2 r}\left(\left|v_{\rho}\right|+\left|v_{z}\right|+\right.$ $\left.g\left|v_{\phi}\right|\right) \sim g v / r$. Here, $v_{\rho}, v_{z}$ and $v_{\phi}$ are the components of the c.m velocity in the cylindrical coordinate and $v$ the speed rate. On the other hand, the lower limit of $\left|E_{ \pm}-E_{0}\right|$ is $\left|\sqrt{\left|\Omega_{c}\right|^{2}+\left|\Omega_{p}\right|^{2}+(V / 2)^{2}}-V\right|$. Then the condition (23) can be rewritten as

$$
\frac{g}{r} \sqrt{\frac{2 E}{M}}<<\frac{1}{2}\left(\sqrt{4 \xi(r+|z|)+E^{2}}-E\right) .
$$

Here we have used the fact that the upper limit of $v$ is $\sqrt{2 E / M}$ and the upper limit of $V$ is the atomic energy $E$.

The adiabatic condition (24) can be satisfied in some realistic cases. For instance, we confider the cesium atoms trapped around the origin $r=0$. We assume $\xi=\pi \times 10^{10} \mathrm{Hzm}^{-1 / 2}$ and the atomic energy $E \sim 10^{-26}$ J. Then it is easy to see that when the optical angular momentum $g \sim 10^{1}$, the adiabatic condition is satisfied when $r>10^{-6} \mathrm{~m}$. Therefore, if the scale of the atomic ensemble is $r \sim 10^{-3} \mathrm{~m}$, the adiabatic approximation holds in almost all the region of the atomic motion. Another case discussed above is that the cesium atoms are trapped around the point $\left(0,0, z_{0}\right)$ by the harmonic potentials. We assume $z_{0} \sim 10^{-3} \mathrm{~m}, g \sim 10^{4}$ and the frequencies of the trap potentials are $\omega_{z} \sim 10^{6} \mathrm{~Hz}$ and $\omega \sim 10^{2} \mathrm{~Hz}$. Then the adiabatic condition can be met again if $\xi$ and $E$ have the same values as mentioned above. Therefore, the induced change of zero point energy $-m g /\left(4 M z_{0}^{2}\right)$ is about $10^{1} \mathrm{~Hz}$. The frequency shift $\widetilde{\omega}-\omega$ caused by the monopole potential will have the same order with $\omega$.
Before concluding this paper we make a remark on the results that we have obtained. As we have shown, when the certain conditions $\left|\Omega_{p}\right|^{2}=\xi(r+z),\left|\Omega_{c}\right|^{2}=\xi(r-z)$ is satisfied, the singularity of the monopole potential can be eliminated by dividing the space into two caps. To see the universality in choosing the profile of light beam we make the following change: $\left|\Omega_{c}\right|^{2}=\xi[(2 \eta-1) r-z]$ where $\eta>1$. In this case, the induced gauge potential will be $\mathbf{A}_{0}=g(1+\cos \theta) \hat{e}_{\phi} /(2 \eta r \sin \theta)$. Thus the singularity of $\mathbf{A}_{0}$ can NOT be eliminated with the above method when $g / \eta$ is not an integer. This is because in this case we will have $\psi_{0 b}(\mathbf{r})=\psi_{0 a}(\mathbf{r}) e^{-i g \phi / \eta}$ and if $g / \eta$ is not an integer, $e^{-i g \phi / \eta}$ is not a single valued function. We notice that a similar situation appears when the Dirac's quantization condition is not satisfied [13]. This concluding remark shows the possibility to test the subtle effect of magnetic monopole with manipulation of ultra cold atoms.

This work is supported by the NSFC and the knowledge Innovation Program (KIP) of the Chinese Academy of Sciences. It is also founded by the National Fundamental Research Program of China with No. 001GB309310. One (CPS) of the authors thanks Y.B. Dai for useful discussions.

${ }^{a}$ Electronic address: suncp@itp.ac.cn

${ }^{b}$ Internet www site: http:// www.itp.ac.cn/ suncp

[1] P. A. M. Dirac, Proc. Roy. Soc. A 133, 60 (1931).

[2] G. 't Hooft, Nucl. Phys. B 79, 276 (1974); A. M. Polyakov, JETP Lett. 20, 194 (1974).

[3] T. T. Wu and C. N. Yang, Phys. Rev. D 12, 384 (1975).

[4] M. V. Berry, Proc. R. Soc. London A 392, 45 (1984); A. Shapere and F. Wilczek (Ed.), Geometric Phases in Physics (World Scientific, Singapore, 1989).

[5] Z. Fang et.al., Science 302, 92 (2003).

[6] J. Moody, A. Shapere, F. Wilczek and A. Zee, Phys. Rev. Lett. 56, 893 (1986); C. A. Mead, Phys. Rev. Lett. 59, 161 (1987).

[7] M. Born, R. Oppenheimer, Ann. Physik 84, 457 (1930).

[8] C. P. Sun, M. L. Ge, Phys. Rev. D 41, 1349 (1990).

[9] S. E. Harris, Physics Today 50, 36 (1997); M. D. Lukin, Rev. Mod. Phys. 75, 457 (2003).

[10] C. P. Sun, Y. Li, and X. F. Liu, Phys. Rev. Lett. 91, 147903 (2003).

[11] L. Allen, M. Padgett, and M. Babiker, Prog. Opt. 39, 291 (1999); L. Allen, S. M. Barnett, and M. J. Padgett, Optical Angular Momentum (Institute of Physics, Bristol, 2003).

[12] G. Juzeliunas and P. Ohberg, cond-mat/0402317.

[13] T. T. Wu and C. N. Yang, Nucl. Phys. B 107, 365 (1976).

[14] I. Tamm, Z. Phys. 71, 141 (1931). 